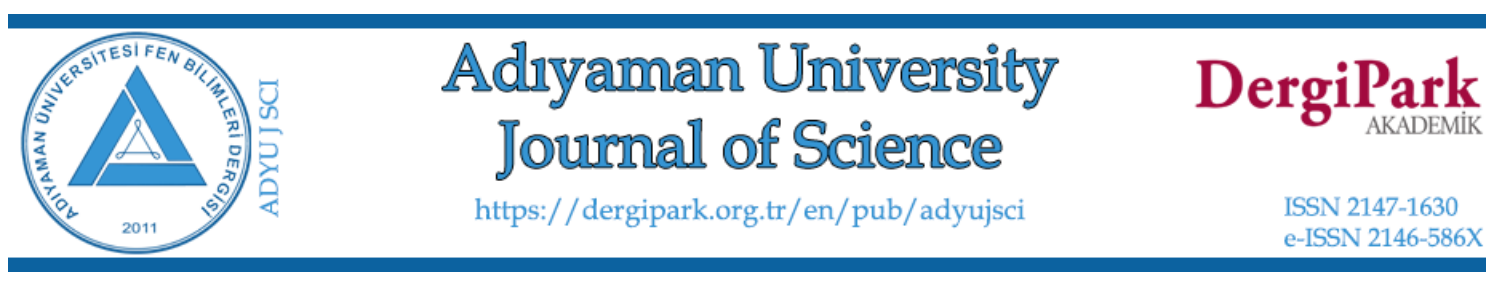

A Probabilistic Atomic Radius Definition: Application to Ground State Hydrogen-

\title{
Like Atoms
}

\author{
Fatih Mehmet AVCU ${ }^{1, *}$, Serkan ALAGÖZ ${ }^{2}$ \\ ${ }^{1}$ Inonu University, Department of Informatics, Malatya, Turkey \\ fatih.avcu@inonu.edu.tr,ORCID:0000-0002-1973-7745 \\ ${ }^{2}$ Inonu University, Department of Physics, Malatya, Turkey \\ serkan.alagoz@inonu.edu.tr,ORCID:0000-0003-2642-8462
}

Received: 25.05 .2020

Accepted: 03.12 .2020

Published: 30.12 .2020

\begin{abstract}
Several atomic radius concepts were proposed to express size of atoms in chemistry and physics. This study briefly surveys widely used definitions of atomic radiuses and introduces a probabilistic atomic radius concept that is based on the probability measure, which considers probability of the outermost electron to be in a spherical volume that is centered by nucleus of a hydrogen-like atom. Depending on this probability measure, $99 \%$ probabilistic atomic radius is calculated for a ground state hydrogen atom and its interpretation for hydrogen-like atomic systems is discussed.
\end{abstract}

Keywords: Quantum mechanics; Solutions of wave equations; Atomic radius; Bound states.

\section{Olasılıksal Atomik Yarıçap Tanımı: Taban Durumu Hidrojen Benzeri Atomlara}

\section{Uygulanması}

$\ddot{O} \mathbf{z}$ 
Kimya ve Fizik alanında atomların büyüklüğünü ifade etmek için birkaç atom yarıçapı kavramı önerilmiştir. Bu çalışmada, atomik yarıçap tanımları incelenmiş, olasılık ölçüsüne dayanan ve en dıştaki elektronun hidrojen benzeri bir atomun çekirdeği ile merkezlenmiş küresel bir hacimde olma olasılı̆̆ını dikkate alan olasılıksal bir atomik yarıçap kavramını sunulmuştur. $\mathrm{Bu}$ olasılık ölçüsüne bağlı olarak, temel durum hidrojen atomu için \%99 olasılık ile atomik yarıçapı hesaplanmış ve bunun hidrojen benzeri atomik sistemler için yorumlanmıştır.

Anahtar Kelimeler: Kuantum Mekaniği; Dalga denklemlerinin çözümleri; Atom yarıçapı; Bağlı durumlar.

\section{Introduction}

Rutherford-Bohr model, also known as Bohr model, have suggested an atom structure that consists of a nucleus and spinning electrons. This model is relatively primitive model of a hydrogen atom compared to quantum wave mechanics models of atoms. Based on experimental measurements, Bohr atom model suggests that mechanical energy related with energy of atomic electrons should be quantized. According to Bohr's model, electron orbiting around nucleus of atom can be certain particular states of motion, which is called "stationary states" [1]. In other words, the model implies that an electron orbits only at certain distances to nucleus depending on energy state of an atom. Such consideration of an atom allows fundamental definition of an atom radius with respect to distances of electron orbits to nucleus in space. For single electron atoms such as hydrogen, the smallest possible orbit with lowest energy state has an orbital radius, which was called Bohr radius. Then, the Bohr radius became an important physical constant associated with atomic scales and it is approximately equal to the most probable distance between the nucleus and the electron in a hydrogen atom in its ground state. Value of Bohr radius is approximately $a_{0}=\frac{\mathrm{h}^{2}}{m k e^{2}}=0.0529 \mathrm{~nm} \cong 0.53 A^{o}$. An important point for Bohr radius is the fact that it is the radial distance to nucleus that has the peak of probability density of an electron [2].

The atomic radius is commonly used in chemistry and physics to assign a size for atoms in spatial domain, and it is generally considered as distance between the nucleus and the boundary of the surrounding cloud of electrons [3]. Experimental value of atomic radius came out in works of X-ray crystallography with a question whether the same radii is valid for atoms of the same element [4]. More experimental data on crystal structures showed that the same atom in different crystal structures could not have the same experimental radii [5].

There is not unique value of atomic radius parameter because it changes depending on the atom's state and the purpose of measurements or analyses [3]. The main difficulty is that 
definition of atomic boundary varies depending on the context of analyses. For example, atomic radius changes depending on several factors: atoms can be isolated or bounded; type of bounding (ionic or covalent) affects atomic boundary considerations and energy states of atoms are effective in atom models. Therefore, several non-equivalent definitions of atomic radius are utilized at various contexts. For ease of atomic radius calculations, atoms were mainly assumed to have a spherical shape and such approximate models are utilized for explanations of several phenomena, such as the density of matter, the diffusion of fluids, arrangement of atoms and ions in crystals and the size and shape of molecules [6]. Such a spherical confinement of hydrogen atom enables investigation of many properties in several studies [7]. A set of empirical atomic radii has been proposed and sum of the atomic radii of two atoms forming a bond in a crystal or molecule is used for estimation of approximate distance between nucleuses of those two atoms [8]. For other atoms and molecules, relationship between atomic radius and chemical properties has been investigated. A recent work indicated existence of a linear relationship between the atomic radius and deposition potentials for lanthanide metals [9]. Another study considered decoding of atomic and ionic radii of transition metals in terms of energy response against changes in electron number. Employing charge sensitivity analysis and the electronegativity equalization principle, authors have presented an interpretation of the electronic structure transformation (electronfollowing/preceding perspectives) into atomic diameters [10].

This study introduces a probabilistic atomic radius concept, which is based on probability of the outermost electrons to be in the spherical volume that is centered by nucleus. Hence, probability measure is used to express tendency of the outermost electron in a nucleus centered, spherical space volume. Cumulative radial probability density of electron, which is obtained from solution of Schrodinger equation for hydrogen atom, is used and the $99 \%$ probability of electron to be existent in this volume is considered for probabilistic atomic radius definition. It is the case that spherical space volume involves $99 \%$ of electron cloud, which infers a $99 \%$ probability of an electron existence in this spherical volume. The $99 \%$ probabilistic atomic radius is defined arithmetically as the radius of such nucleus centered spherical volume that involves the valance electron with a probability of $99 \%$.

\section{Brief Survey of Atomic Radius}

This section summarizes commonly used atomic radius definitions:

Van der Waals radius is the half of minimum distance between the nuclei of two atoms that are not bound to the same molecule [11]. It is widely used to calculate Van der Waals volume of 
atoms. For hydrogen molecules, value of Van der Waals radii is found $0.12 \mathrm{~nm}$. Neutron diffraction experiments can provide an estimate experimental value of Van der Waals radius.

Ionic radius defines a radius for an ion in ionic crystals. Sum of ionic radiuses is utilized to estimate gaps between two adjacent oppositely charged ions [11]. Ions were assumed to be hard spherical bodies with ionic radii. The sum of ionic radii is used to estimate the distance between cations and anions in a crystal lattice. X-ray crystallography is used to estimate ionic radius of ions. Hydrogen ions lose their single electrons and they become a positive charged ion formed only by a proton nucleus without any electron. Therefore, ionic radius is not applicable for hydrogen ions so that it does not contain a valance electron to form space volume.

Covalent radius is the radius that is defined for covalently bounded atoms [6]. The length of covalent bond is estimated by the sum of covalent radii [11]. For Hydrogen with single bond, covalent radius is estimated to be $0.038 \mathrm{~nm}$. X-ray diffraction and rotational spectroscopy are used to estimate experimental value of covalent radius.

Metallic radius is the half distance between the two adjacent metal ions in a metallic structure [6]. It is defined for atoms that can be joined by metallic bonds.

Bohr radius is considered as the most probable distance between the nucleus and the electron in a hydrogen atom in its ground state. Value of Bohr radius was given $a_{0} \cong 0.0529 \mathrm{~nm}$ and depending on energy states, radius of hydrogen atom is written by $r_{n}=a_{o} n^{2}$, where principle quantum number $n$ takes 1,2,3. Bohr radius of hydrogen atom is considered as a physical constant to scale atomic distances [2].

Critical cage radius is considered according to binding energy of ground state hydrogen atom. A spherical box is assumed to have impenetrable surface and the radius of sphere box reduced until binding energy diminishes $[12,13]$. A critical value of the sphere radius at which the binding energy becomes zero is called critical cage radius $[7,12,13]$. Binding energy of ground state of the hydrogen atom is calculated as a function of the sphere radius [13]. For hydrogen in ground state, critical cage radius is found about $1.83 a_{0}=0.097 \mathrm{~nm}$ [7].

Another probabilistic modeling of atom radius was given corresponding to the maximum of the radial probability function of the valence electron [14-16]. For a hydrogen-like atom, peak of radial probability function is exactly at Bohr radius [14-16]. On the other hand, expected value of radial probability function is accounted as atomic radii. For ground state hydrogen atoms, expected value is calculated as $\langle r\rangle=1.5 a_{0}=0.0794 \mathrm{~nm}[2]$. 


\section{Volumetric Probability Distribution of a Particle in Quantum Mechanics}

As known, electrons in the hydrogen atom have a spherically symmetric potential, therefore, spherical polar coordinates are used for the time independent solution of Schrodinger wave equation. Schrodinger equation is generally solved for the hydrogen atoms by separating the variables in spherical polar coordinate system as follows [2, 17-19],

$$
\psi_{n, l, m_{l}}(r, \theta, \varphi)=R(r) P(\theta) F(\varphi)
$$

where, parameters $(r, \theta, \varphi)$ are components of polar coordinate. Schrodinger equation is generally expressed as [2, 17-19],

$$
\begin{aligned}
& \frac{-\hbar^{2}}{2 \mu} \frac{1}{r^{2} \sin \theta}\left[\sin \theta \frac{\partial}{\partial r}\left(r^{2} \frac{\partial \psi}{\partial r}\right)+\frac{\partial}{\partial \theta}\left(\sin \theta \frac{\partial \psi}{\partial \theta}\right)+\frac{1}{\sin \theta} \frac{\partial^{2} \psi}{\partial \theta^{2}}\right]+U(r) \psi(r, \theta, \varphi) \\
& =E \psi(r, \theta, \varphi)
\end{aligned}
$$

where, the potential energy is $U(r)=-e^{2} / 4 \pi \varepsilon_{0} r$ and the parameter $E$ denotes energy of system. The reduced mass is written as $\mu=m_{e} m_{p} /\left(m_{e}+m_{p}\right)$. Solution of this equation is found depending on states of principle quantum number $(n)$, orbital quantum number $(l)$, magnetic quantum number $\left(m_{l}\right)$.

In quantum mechanics, the maximum of square of wave function shows the most likely position where a particle can exist in space [2,17-19]. Probability of a free particle in a range $x \in$ $\left.\left[x_{1}, x_{2}\right]\right)$ is expressed as,

$$
P\left(x_{1} \leq x \leq x_{2}\right)=\int_{x_{2}}^{x_{2}}|\psi(x)|^{2} d x
$$

where, $\psi(x)$ is normalized wave function of the particle, which satisfies the condition $\int_{-\infty}^{\infty}|\psi(x)|^{2} d x=1$ to express a probability density function on space. This condition also infers that the particle definitely exist in an infinite space. The radial probability density is defined as multiplication of the normalized wave function square and a spherical shell volume element $4 \pi r d r$ as,

$$
d P=|\psi(r)|^{2} 4 \pi r d r
$$

When a finite volume of spherical space element, which is bounded in radial range of $r_{1} \leq$ $r \leq r_{2}$, is considered, volumetric probability density of a particle can be expressed as, 


$$
P\left(r_{1} \leq r \leq r_{2}\right)=\int_{r_{2}}^{r_{2}}|\psi(r)|^{2} 4 \pi r d r
$$

where, $r$ is the diameter of spherical shell volume. Then, one can express volumetric probability density function of the radial distance $r$ for a particle that is confined into spherical space volume with radius $r$ as,

$$
P(r)=\int_{0}^{r}|\psi(r)|^{2} 4 \pi r d r
$$

This function expresses cumulative radial density function of a valance electron.

\section{Probabilistic Atom Radius According to Orbiting Electron Probability}

In quantum mechanics, one can mention probability of a particle to exist in space. Since atoms are composed of subatomic particles, probabilistic atomic radius is preferable to consider a spherical volume of atoms, where all building blocks or elements of an atom exist with a high probability in this volume. Even though radial probability density infinitely overlay throughout space, a finite space volume can be represented by a probabilistic boundary, which infers a space volume with a high probability of containing all elements of Bohr atom model (nucleus and electrons). A probability measure of $q \in(0,1)$ can be used to express such probabilistic boundaries or probabilistic confinement of space volumes of an atom. The probability measure $q$ excludes the value of 0 because zero probability infers absence of electrons in space, and it also excludes the value of 1, which infers a definite existence of all elements of atom; however, it is only possible for infinite (unbounded) space volume with an infinite radius in quantum mechanical point of view.

The $q \%$ probabilistic atomic radius $\left(r_{q}\right)$ represents a finite spherical volume around the nucleus of atom, which contains the outermost electron with a probability value of $q \%$. Probabilistic atom radius $r_{q}$ can be calculated by solving the equation $P\left(r_{q}\right)=q$, which can be expressed by using cumulative radial density function of orbital electrons as

$$
\int_{0}^{r_{x}}|\psi(r)|^{2} 4 \pi r d r=q
$$

This paper particularly considers $99 \%$ probabilistic radius $\left(r_{0.99}\right)$ of hydrogen atom and presents an analysis for atomic radius and volume in quantum mechanical perspective. In order to calculate $99 \%$ probabilistic radius, the equation of $P\left(r_{0.99}\right)=0.99$ is solved and $r_{0.99}$ radius is 
calculated, numerically. Fig. 1 depicts $r_{0.99}$ probabilistic radius of an atom based on electron probability. In the spherical volume with $r_{0.99}$, valance electron exists with a probability of $99 \%$.

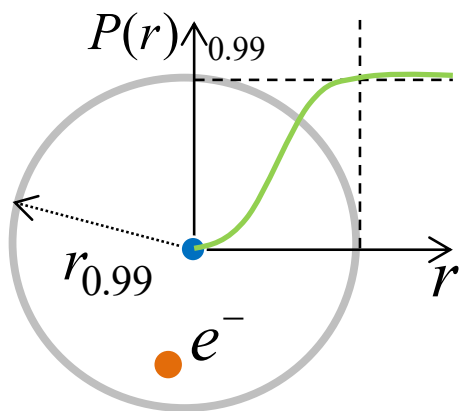

Figure 1: $r_{0.99}$ probabilistic radius of an atom

\section{Probabilistic Atomic Radius $\left(r_{0.99}\right)$ for Single Electron Hydrogen-like Atoms}

The radial probability density of an electron for ground state hydrogen atom (the quantum numbers are $n=1, l=0, m_{l}=0$ for $1 \mathrm{~s}$ state) is written by multiplying the square of the normalized wave function, given by

$$
\psi_{n, l, m_{l}}(r, \theta, \varphi)=R(r) P(\theta) F(\varphi)=\frac{2 e^{-r / a_{0}}}{a_{0}^{3 / 2}} \frac{1}{\sqrt{2}} \frac{1}{\sqrt{2 \pi}}=\frac{e^{-r / a_{0}}}{\sqrt{\pi} a_{0}^{3 / 2}}
$$

$[2,17-19]$ and the spherical shell volume element $4 \pi r^{2}$ as follows:

$$
d P=\left[\frac{e^{-r / a_{0}}}{\sqrt{\pi} a_{0}^{3 / 2}}\right]^{2} 4 \pi r^{2} d r=\frac{4}{a_{0}^{3}} r^{2} e^{-2 r / a_{0}} d r
$$

For the probability of electron to be in the spherical volume, the following cumulative radial density function for existence of orbital electron in spherical volume surrounding a hydrogen nucleus is written according to Eqn. (6) as

$$
P(r)=\int_{0}^{r}\left[\frac{e^{-r / a_{0}}}{\sqrt{\pi} a_{0}^{3 / 2}}\right]^{2} 4 \pi r^{2} d r
$$

where parameter $r$ is radial distance to nucleus of atom. The solution of Eqn. (10) is written by,

$$
P(r)=1-\frac{1}{a_{0}^{2}}\left(a_{0}^{2}+2 a_{0} r+2 r^{2}\right) e^{-2 r / a_{0}}
$$


where, $a_{0}$ is an atomic unit length, also known as Bohr radius. It is found as $a_{0}=\frac{\hbar^{2}}{m k e^{2}}=$ $0.0529 \mathrm{~nm} \cong 0.53 A^{o}$ [1]. $99 \%$ probabilistic atom radius $\left(r_{0.99}\right)$ was defined as the radius of a spherical cage of the nucleus of hydrogen atom, where the electron is found with a probability of $99 \%$. To calculate $r_{0.99}$, the following equation is written by consideration of $P\left(r_{0.99}\right)=0.99$.

$$
1-\frac{1}{a_{0}^{2}}\left(a_{0}^{2}+2 a_{0} r_{0.99}+2 r_{0.99}^{2}\right) e^{-2 r_{0.99} / a_{0}}=0.99
$$

This equation can be numerically solved by using the code that is shown in Fig. 2. If numerical calculation is performed by $\Delta r$ unit increment, error in calculations becomes less than $\Delta r$.

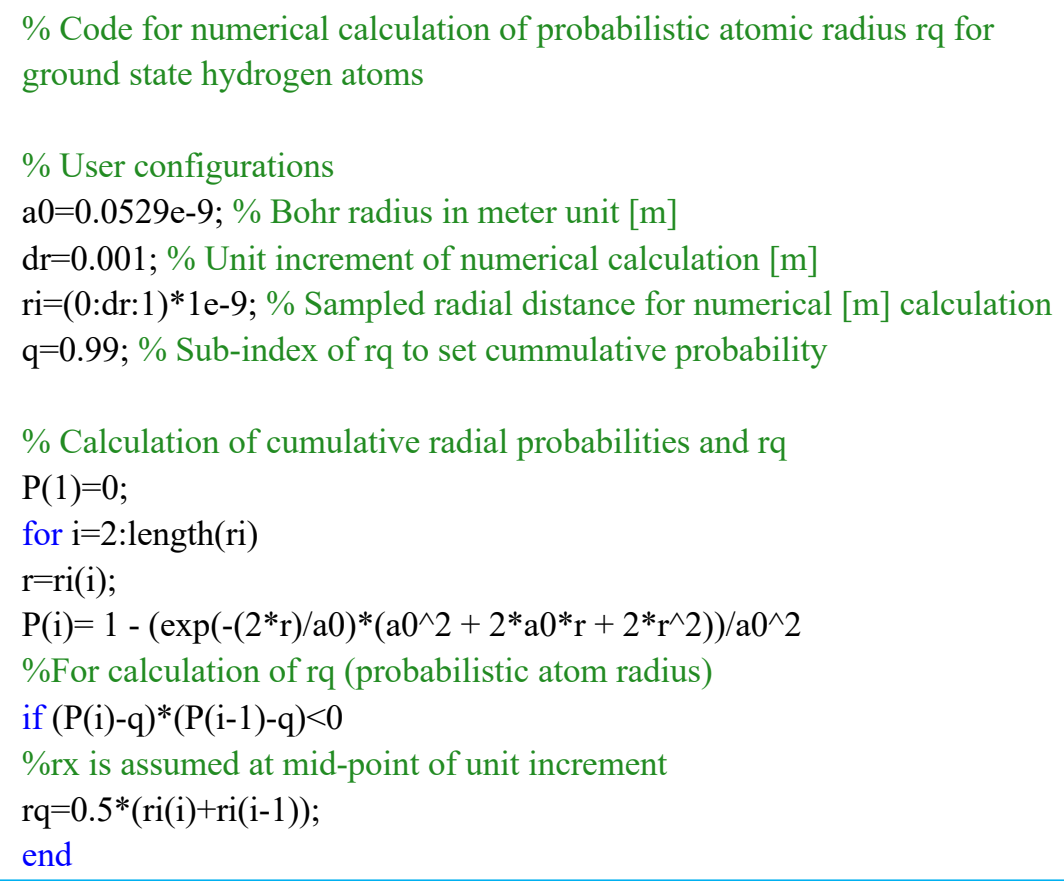

Figure 2: Proposed code for numerical calculation of equation $r_{q}$ for $q=0.99$

Fig. 3 shows volumetric probability of electron in ground state (1s) hydrogen atom. According to proposed definition, $P\left(r_{0.99}\right)=0.99$ is numerically solved and the $99 \%$ probabilistic atomic radius for isolated, ground state hydrogen atom is found $r_{0.99} \cong$ $2.2510^{-10} \mathrm{~m}$, which is about $4.2 \mathrm{Bohr}$ radius $\left(4.2 a_{0}\right)$. In general, hydrogen atom is assumed to have one Bohr radius $\left(a_{0}\right)$ and it coincides to an electron probability of $32 \%\left(P\left(r_{0.32}\right)=0.32\right)$. 


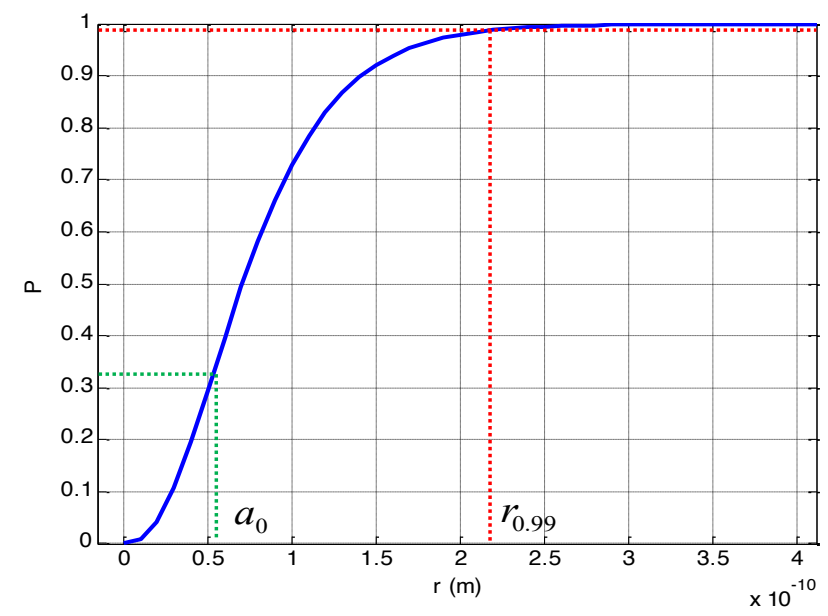

Figure 3: Cumulative probability density of electrons in spherical volume

Fig. 4 shows radial probability density function of ground state electron. The figure shows Bohr radius and the expected value of radial distance $\langle r\rangle=3 a_{0} / 2$ that is 1.5 times the Bohr radius. Bohr radius appears at a maximum of radial density function. As seen in figures, $r_{0.99} \cong$ $4.2 a_{0}$ includes a large portion of radial probability density and implies a high probable inclusion of an electron by a spherical volume. The existence probability of the outermost element of atom, namely the valance electron probability, is considered. Bohr radius or expected value of radial distance does not imply a volume with such a high probable of electron existence. Fig. 5 shows two-dimensional (2D) view of atomic volumes in spatial domain, which are drawn corresponding to $r_{0.99}$ and $a_{0}$ radiuses. When compared, $r_{0.99}$ radii better represents size of atom in term of quantum mechanical aspects.

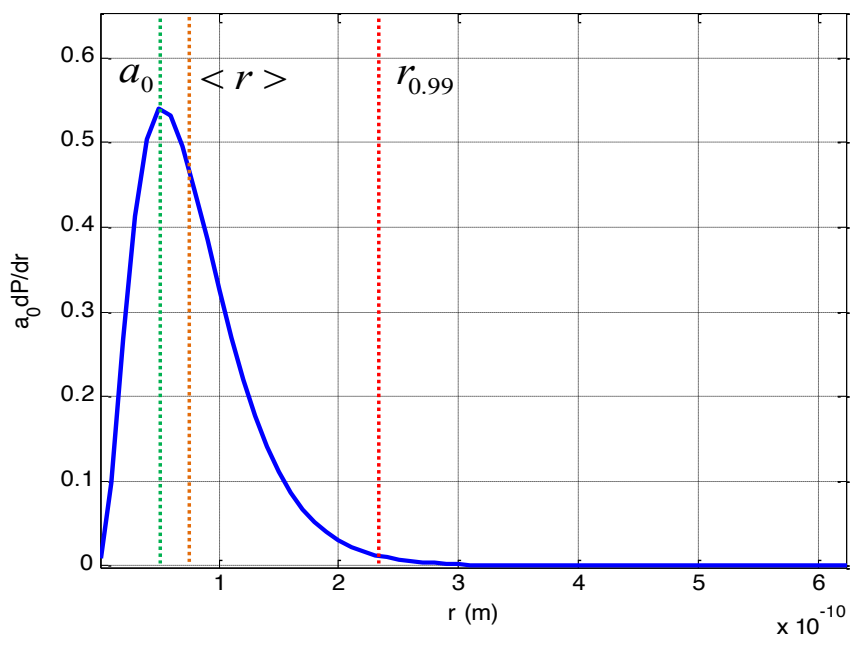

Figure 4: Radial probability density of hydrogen atom and some atomic radiuses 

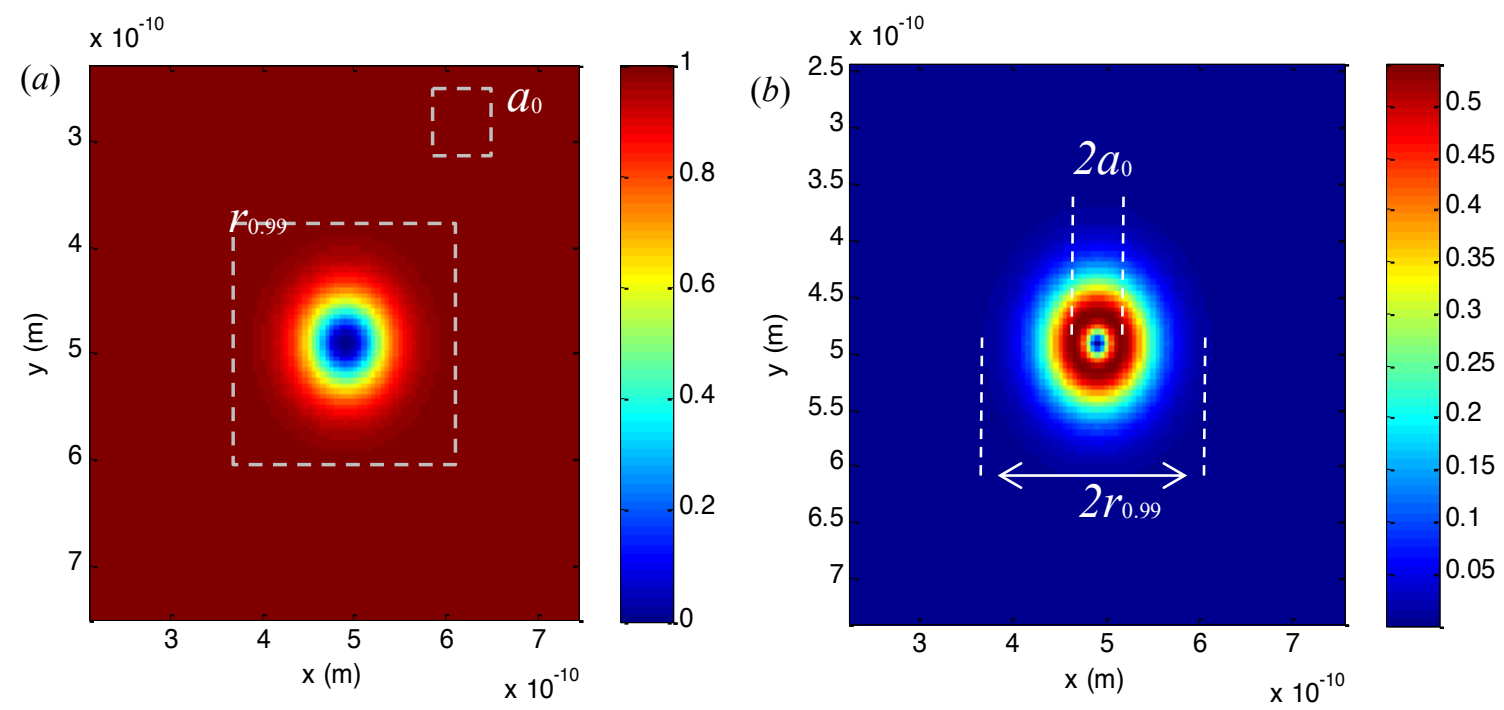

Figure 5: (a) 2D view of cumulative probability density of electrons in spherical volume (b) 2D view of radial probability density of hydrogen atom and comparisons of corresponding atomic radiuses

Table 1 compares values of several atomic radius definitions that can be applicable for hydrogen atoms. The $r_{0.99}$ probabilistic atomic radius has the largest value that ensures all fundamental elements (electron and proton) of a ground state hydrogen atom are included with a probability of 0.99 within the spherical space volume. The classes of $q \%$ probabilistic atomic radiuses $\left(r_{q}, q \in(0,1)\right)$ can be particularly useful when analyzing and modeling quantum mechanical interaction among atoms and expressing their results. This definition can embody an atom in space with its quantum mechanical entity. Besides $r_{0.99}$ radius, Van der Waals radius and Critical cage radius can express a volume of Hydrogen atom with high possibilities of electron existence, which are $83 \%$ for Van der Waals radius and $70 \%$ for Critical cage radius, respectively.

Table 1: Values of several atomic radius definitions, which are applicable for hydrogen atom

\begin{tabular}{llll}
\hline Atomic Radius Definitions & $\begin{array}{l}\text { Values in } \\
\mathbf{n m}\end{array}$ & $\begin{array}{l}\text { Normalized } \\
\text { values } \\
(\mathbf{B o h r} \text { radius } \\
\left(\boldsymbol{a}_{\mathbf{0}}\right)\end{array}$ & $\begin{array}{l}\text { Their correspondence } \\
\text { to probabilistic } \\
\text { atomic radii } \\
\left(\mathbf{q} \%, \boldsymbol{r}_{\boldsymbol{q}}\right)\end{array}$ \\
\hline $\begin{array}{l}\text { Van der Waals radius } \\
\begin{array}{l}\text { Covalent radius } \\
\text { Pritical cage radius } \\
\text { (Maximum of radial probability density) }\end{array}\end{array}$ & 0.12 & 2.26 & $\left(83 \%, r_{0.83}\right)$ \\
$\begin{array}{l}\text { Expected value of radial probability } \\
\text { density }\end{array}$ & 0.038 & 0.71 & $\left(17 \%, r_{0.17}\right)$ \\
\hline $\mathbf{9 9 \%}$ probabilistic atomic radius $\left.\boldsymbol{(}_{\mathbf{0 . 9 9}}\right)$ & 0.0794 & 1.5 & $\left(70 \%, r_{0.70}\right)$ \\
\hline & 0.225 & 4.2 & $\left(57 \%, r_{0.57}\right)$ \\
\hline
\end{tabular}

\section{Normalization of Spatial Domain according to Radial Probability Factor of Atoms}

Another useful application of probabilistic radial distance $r_{0.99}$ is that, space surrounding nucleus of atom can be mapped to radial probability factor $q \in(0,1)$ that allows a normalization 
of real distances according to cumulative radial probability of electrons. One of the advantages of this normalization is that it can compensate difficulties in analyses, which are associated with diversity in atomic radius. Use of radial probability factor $q \in(0,1)$ instead of spatial distance metrics allows consideration of a limited-range and common factor $q \in(0,1)$ for analysis of all atoms.

Normalization of spatial radial distance for $q$ radial probability factor of hydrogen atom can be expressed as $d(q)=r / r_{q}$. Normalization of a spherical space volume can be expressed as $V(q)=\left(r / r_{q}\right)^{3}$. In this case, atom density can be normalized according to the electron probability as

$$
\rho(q)=n / V(q)
$$

where $n$ is the number of atoms in the normalized volume $V(q)$ that is for probability factor $q$. Accordingly, one can analyze interaction between atoms on the bases of radial probability factor $q \in(0,1)$.

\section{Conclusion}

This study introduces a probabilistic atomic radius model that represents an atom with a nucleus centered spherical space volume according to cumulative radial probabilities of a valance electron. The $r_{0.99}$ probabilistic atomic radius expresses a spherical atom volume that is the most likely to observe a valance electron. This assumes the case that an electron can be found in this spherical volume with $99 \%$ probability. For ground state Hydrogen atom, atomic radius $r_{0.99}$ is calculated and its value is compared with values of other substantial atomic radius definitions.

Radius $r_{q}$ definition allows sizing an atom in a space volume according to probability space of the time independent solutions of Schrödinger wave equation. Particularly, this probabilistic radius concept can contribute to analyze quantum mechanical interactions among atoms depending on a probabilistic radial dimension $r$, and this atomic radius concept can project the probability space to the real spatial space of atom.

Future studies can address calculation of $r_{0.99}$ values for higher quantum numbers and energy states of hydrogen atoms. While compressing hydrogen atoms, effects of interactions between atoms can be observed experimentally. In analysis of experimental results, changes in phase or properties of various atoms can be elaborated on the bases of probability factor $q$. 


\section{References} 2013.

[1] Zettili, N., Quantum mechanics: concepts and applications, 2nd ed., 408 pp, Wiley,

[2] Adamson, A.W., Advanced inorganic chemistry. By F.A. Cotton and G. Wilkinson, Inorganic Chemistry, 2 (3), 665-665, 1963.

[3] Bragg, W.L., The arrangement of atoms in crystals, Nature, 106 (2675), 725-725, 1921.

[4] Wyckoff, R.W.G., On the hypothesis of constant atomic radii, Proceedings of the National Academy of Sciences of the United States of America, 9 (2), 33-38, 1923.

[5] Huggins, M.L., Atomic radii I, Physical. Review, 19 (4), 346-353, 1922.

[6] Michels, A., De Boer, J., Bijl, A., Remarks concerning molecural interaction and their influence on the polarisability, Physica, 4 (10), 981-994, 1937.

[7] Montgomery, H.E., Sen, K.D., Electron density and its derivatives at the nucleus for spherically confined hydrogen atom, International Journal of Quantum Chemistry, 109 (4), 688 692, 2009.

[8] Xu, H., Qu, J., Zhang, M., Yan, Y., Sun, X., Zheng, Y., Qiu, M., Liu L., The linear relationship derived from the deposition potential of $\mathrm{Pb}-$ Ln alloy and atomic radius, New Journal of Chemistry, 42 (20), 16533-16541, 2018.

[9] Szarek, P., Witkowski, M., Woźniak, A.P., Unconventional look at the diameters of quantum systems: could the characteristic atomic radius be interpreted as a reactivity measure, The Journal of Physical Chemistry C, 123 (18), 11572-11580, 2019.

[10] French, S.J., Nature of the chemical bond. second edition, revised (Pauling, Linus), Journal of Chemical Education, 17 (11), 551, 1940.

[11] Sommerfeld, A., Welker, H., Künstliche Grenzbedingungen beim Kepler problem, Annalen der Physik, 424 (1-2), 56-65, 1938.

[12] Varshni, Y.P., Critical cage radii for a confined hydrogen atom, Journal of Physics B: Atomic, Molecular and Optical Physics, 31 (13), 2849-2856, 1998.

[13] Gill, P.M.W., Johnson, B.G., Pople, J.A.A., Standard grid for density functional calculations, Chemical Physics Letters, 209 (5), 506-512, 1993.

[14] Gill, P.M.W., Johnson, B.G., Pople, J.A., Two-electron repulsion integrals over gaussian s functions, International Journal of Quantum Chemistry, 40 (6), 1991.

[15] Slater, J.C., Atomic shielding constants, Physical Review, 36 (1), 57-64, 1930.

[16] Tang, C.L., Fundamentals of quantum mechanics: for solid state electronics and optics, Cambridge University Press, 224 pp, 2005.

[17] Griffiths, D.J., Schroeter, D.F., Introduction to quantum mechanics 3rd ed., Cambridge University Press, 495 pp, 2018.

[18] Rogers, G.L.A., Visual demonstration of the simple quantum theory of the hydrogen atom, European Journal of Physics, 15 (3), 110-110, 1994. 